\title{
La Competencia Digital Docente. El caso de las universidades andaluzas
}

\author{
Julio Cabero-Almenara \\ Universidad de Sevilla, Sevilla, España \\ mail: caberolus.es \\ ORCID: https://orcid.org/0000-0002-1133-6031
}

\section{Julio Barroso-Osuna}

Universidad de Sevilla, Sevilla, España

mail: jbarrosolus.es

ORCID: https://orcid.org/0000-0003-0139-9140

\section{Margarita Rodríguez-Gallego}

Universidad de Sevilla, Sevilla, España

mail: margaguezdus.es

ORCID: https://orcid.org/0000-0001-6959-4829

\author{
Antonio Palacios-Rodríguez* \\ Universidad de Sevilla, Sevilla, España \\ mail: aprodriguezQus.es \\ ORCID: https://orcid.org/0000-0002-0689-6317
}

\section{RESUMEN}

La revolución digital ha transformado los modos de hacer, actuar y formar en el ámbito universitario. Por ello, el fomento y desarrollo la competencia digital docente (CDD) en Educación Superior es un aspecto clave en el siglo XXI. El propósito de este estudio es conocer el nivel de competencia digital del profesorado universitario andaluz, así como identificar si existen diferencias significativas entre la autopercepción antes y después de realizar el cuestionario. Para ello, se plantea un diseño no experimental con enfoque descriptivo y contraste de hipótesis. La muestra objeto de estudio queda conformada por un total de 2262 docentes pertenecientes a las 9 universidades públicas de Andalucía (España), que contestan el instrumento DigCompEdu Check-In adaptado al contexto español. En términos generales, los resultados muestran que el nivel es moderado. En concreto, las áreas mejor valoradas han sido las de Pedagogía Digital y Recursos Digitales. Así mismo, se demuestra que la autopercepción antes de realizar el cuestionario es superior a la realizada en un momento posterior. Estos datos explican el fenómeno denominado como idealización competencial. Por este motivo, se recomienda llevar a cabo planes personalizados de formación docente avalados por marcos sólidos como DigCompEdu.

Palabras clave: competencia digital, enseñanza universitaria, DigCompEdu, formación del profesorado.

\section{Digital Competence for Educators. The case of Andalusian universities}

\begin{abstract}
The digital revolution has changed the ways of doing, acting and training at the university. For this reason, the development of digital teaching competence (DTC) in Higher Education is a key aspect of 21st century. The purpose of this study is to know the level of digital competence of the Andalusian professor, as well as to study his self-perception before and after his reflection on the DTC. A non-experimental design is planted with a descriptive and contrast of hypotheses model. The object of the study was made up of 2262 professors belonging to the 9 public universities in Andalucía (Spain), who answer the DigCompEdu Check-In instrument adapted to the Spanish context. In general terms, the results show that the level is moderate. Specifically, the most highly valued areas have been those of Digital Pedagogy and Digital Resources. Likewise, self-perception shown before carrying out the survey is higher than the values once it has been answered and reflected on the DTC. These data explain the phenomenon called competence idealization. For this reason, it is recommended to carry out personalized teaching training plans endorsed by solid frameworks like DigCompEdu.
\end{abstract}

Keywords: digital competence, higer education, DigCompEdu, teacher training.

ISSN: 0210-2773

DOI: https: / /doi.org/10.17811/rifie.49.4.2020.363-372 


\section{Introducción}

La revolución digital está llevando a empresas, instituciones y profesionales a una transformación profunda y un cambio radical en sus modos de hacer, actuar y formar. Esta revolución tecnológica ha afectado a todos los sectores de nuestra sociedad, entre ellos el educativo. La Comisión Europea (2012, p. 10), considera que "la revolución digital ha abierto grandes oportunidades para mejorar la calidad, la accesibilidad y la equidad de la educación" al permitir aprender en cualquier momento y lugar y reducir las barreras sociales. Las universidades no escapan a esta realidad, y deben adaptarse a este escenario derivado de los avances tecnológicos.

Las instituciones educativas deben tener políticas y proyectos formativos para no dejar al margen las posibilidades de las tecnologías y trabajar en la formación de la competencia digital. En este sentido, las políticas europeas actúan en una doble dirección; por un lado, en la formación permanente sobre tecnología digital de sus miembros (Consejo de la Unión Europea, 2003) y, por otro, la necesidad de incorporarlas al mercado laboral (Comisión de las Comunidades Europeas, 2003). Para ello, se adoptan ocho competencias clave para el aprendizaje permanente, definidas en la UE como "una combinación de conocimientos, capacidades y actitudes adecuadas al contexto" (Consejo de la Unión Europea, 2018, p. 7). Una de estas competencias es la digital, definida como aquella que "entraña el uso seguro y crítico de las tecnologías de la sociedad de la información para el trabajo, el ocio y la comunicación" (Consejo de la Unión Europea, 2018, p. 9).

Las predicciones indican que la Cuarta Revolución Industrial, en la que nos encontramos, exige destrezas digitales en los empleos futuros (Williamson, Potter y Eynon, 2019). Para ello, la vida social, política, económica y educativa de los países requiere nuevas formas de organización y, por consiguiente, profesionales con una amplia variedad de competencias, entre ellas, la competencia digital (Marín-Díaz, Reche y Maldonado, 2013). Las instituciones universitarias se enfrentan actualmente al desafío de encontrar nuevas formas de desarrollar los procesos de enseñanza-aprendizaje, considerando los cambios tecnológicos, económicos y sociales que se están produciendo (OpenMind BBVA, 2017; Gómez-Parra y Huertas-Abril, 2019, Ruíz Mezcua, 2019). En este sentido, todos los ciudadanos deben desarrollar la competencia digital como una competencia clave del aprendizaje permanente, facilitando la realización personal y el desarrollo, empleabilidad, inclusión social y ciudadanía activa (Consejo de la Unión Europea, 2018).

La competencia digital es definida como aquel conjunto de "habilidades, conocimiento y actitudes que hacen que los alumnos usen los medios digitales para participar, trabajar y resolver problemas, de forma independiente y en colaboración con otros en un momento crítico, responsable y de manera creativa" (Hatlevik, Gudmundsdóttir y Loi, 2015, p. 346). Aunque este término se refiere a la competencia de los alumnos, su desarrollo en el sistema educativo requiere la implementación de la competencia digital por parte del personal docente para poder desempeñar su profesión de una manera eficaz (UNESCO, 2004; Wilson, et al., 2011). La Competencia Digital Docente (en adelante CDD) es un requisito del perfil profesional docente que le permite diseñar, implementar y evaluar acciones formativas orientadas para que el docente utilice de forma didáctica la tecnología con sus estudiantes. Se debe tener muy claro que la Competencia Digital Docente está en constante evolución, al igual que la tecnología (Kluzer et al., 2018).

Los docentes deben poseer una CDD significativa para el dominio de las Tecnologías de la Información y la Comunicación (TIC) y su integración en los procesos de enseñanza aprendizaje (Hatlevik et al., 2018; Roig-Vila, Mengual-Andrés y Quinto-Medrano, 2015), distintas en muchos aspectos del uso que se le puede dar en un ambiente doméstico (Blau y Shamir-Inbal, 2017; Solís de Ovando y Jara, 2019). Al mismo tiempo, el dominio de la CDD faculta al docente no solo para para apoyar las prácticas existentes sino más bien para transformarlas (Uerz, Volman y Kral, 2018). En definitiva, la CDD está relacionada con todas aquellas habilidades, actitudes y conocimientos requeridos por los docentes en la Sociedad del conocimiento y su uso desde una perspectiva didáctico-pedagógica (Cabero-Almenara, Estrada-Vidal y Gutiérrez-Castillo, 2017; García Tartera, 2016).

Además, se destacan algunos factores que se presentan como variables movilizadoras de la CDD teniendo en cuenta su significación y que serán utilizados en el proceso metodológico de esta investigación. Entre ellos, la formación del docente, referida a la experiencia laboral, la formación inicial, y grado de conocimiento de herramientas TIC por el docente (García-Valcárcel MuñozRepiso et al., 2015; Fernández-Márquez, Leiva-Olivencia y López-Meneses, 2017; Beneyto-Seoane y Collet-Sabé, 2018; Bernal, Gonzávez, Hernando y Masanet, 2019; Pozos y Tejada, 2018). En relación con los recursos, calidad de la infraestructura y disponibilidad de dispositivos digitales y tecnologías necesarias (Cela-Ranilla et al., 2017; Gil-Flores, Rodríguez-Santero, y TorresGordillo, 2017). Sobre el tiempo de uso, dedicación al uso de las TIC fuera y dentro del aula como elemento favorecedor de la competencia digital docente (Fernández-Márquez y LeivaOlivencia, 2017). De la misma forma, la falta de tiempo disponible para preparar las sesiones mediante las tecnologías como elemento detractor (Hilliger et al., 2020). Y la referida a la actitud hacia la tecnología, actitudes y creencias que el docente tenga respecto a las posibilidades de las TIC. Se concibe como una variable crítica que determinará la incorporación por el docente de las TIC a la práctica educativa, formas en las cuales son introducidas y las funciones que a las mismas le asignará (Aslan y Zhu, 2016; Bahçivan, Güneş y Üstündağ, 2019; Fernández-Márquez et al., 2017; Choi, Cristol, y Gimbert, 2018; Ghomi y Redecker, 2019; Scherer y Siddiq, 2019). Tal actitud se percibe en la utilización que los docentes realizan de ciertas tecnologías como las redes sociales (Choi et al., 2018; Peñalva Vélez, Fraile y Lacambra, 2018).

Ahora bien, para la adquisición de la CDD, se han propuesto a nivel institucional una serie de marcos competenciales en los cuales deben formarse los docentes. Todos ellos persiguen reconocer la manera en que deben utilizarse e integrarse las tecnologías en la enseñanza, identificar necesidades formativas y proponer itinerarios formativos personalizados (Flores-Lueg y Roig Vila, 2016). Los marcos competenciales más consolidados son el Marco Europeo de Competencia Digital del profesorado DigCompEdu; Estándares ISTE para Educadores; Marco UNESCO de Competencia TIC para Docentes; Marco Común español de Competencia Digital Docente; Marco británico de Enseñanza Digital; Competencias TIC para el desarrollo profesional docente colombiano; Competencias y Estándares TIC para la profesión docente chilena (Cabero-Almenara, Barroso-Osuna, Palacios-Rodríguez y Llorente-Cejudo, 2020; Cabero y Palacios, 2020; Cabero y Martínez, 2019; Durán, Gutiérrez y Prendes, 2016; Lázaro, Usart y Gisbert, 2019; Rodríguez-García et al., 2019). De otro lado, un estudio de evaluación a través del coeficiente competencia experta destaca el modelo DigCompEdu como el más adecuado para evaluar la Competencia Digital Docente (Cabero-Almenara et al., 2020). Dicho modelo fue publicado por el Joint Research Centre de la Comisión Europea (Redecker y Punie, 2017).

El Marco europeo para la competencia digital de los educadores DigCompEdu (Redecker y Punie, 2017) detalla 22 compe- 
tencias digitales organizadas en seis áreas. El área primera compromiso profesional describe el uso eficiente y apropiado de las tecnologías para la comunicación y colaboración con colegas, estudiantes, padres y agentes de la comunidad educativa. Los recursos digitales del área 2 se centran en la selección, creación, modificación y gestión de recursos educativos digitales. Esto también incluye la protección de datos personales de acuerdo con la normativa de protección de datos y el cumplimiento de los derechos de autor al modificar y publicar recursos digitales. La tercera área pedagogía digital se ocupa de la planificación, diseño y uso de tecnologías digitales en práctica docente. Se centra en la integración de recursos digitales y métodos para promover procesos de aprendizaje colaborativos y autorregulados y para guiar estas actividades transformando la enseñanza desde procesos dirigidos el profesorado hasta procesos centrados en el alumno. La evaluación del área cuatro aborda el uso de tecnologías digitales para analizar y evaluar el rendimiento de los estudiantes de manera integral y proporcionar retroalimentación dirigida y oportuna a los estudiantes. El área cinco empoderar a los estudiantes enfatiza la importancia de crear actividades de aprendizaje y experiencias que aborden necesidades de los estudiantes y la participación activa en su proceso de aprendizaje. El área 6, facilitar la competencia digital de los estudiantes en el ámbito de la ciudadanía activa para promover la información y la alfabetización mediática e integrar actividades específicas referidas a la resolución de problemas, creación de contenido digital y uso de tecnología para comunicación y cooperación. Cada competencia individual de DigCompEdu se describe a lo largo de seis niveles competenciales (A1, A2, B1, B2, C1 y C2). Esta propuesta está considerada en la Agenda de Competencias para Europa (Consejo de la Unión Europea, 2018) y en la Iniciativa de la Estrategia Europa 2020 relativa a las Nuevas Capacidades para Nuevos Empleos (Cuartero, Espinosa y Porlán, 2019; Kluzer et al., 2018). En el caso español, la Consejería de Economía, Conocimiento, Empresas y Universidad de la Junta de Andalucía ha creado una plataforma de autodiagnóstico para conocer el nivel de competencias digitales para mejorar la formación a través de un itinerario formativo \#PRODIG (Consejería de Educación y Deporte, 2018). Del mismo modo, DigCompEdu es compatible con el Marco Común de Competencia Digital Docente, publicado por el INTEF, en sus distintas dimensiones y niveles (Cuartero, Porlán y Espinosa, 2016; INTEF, 2017b, 2017a).

DigCompEdu es utilizado como base para desarrollar una herramienta de autopercepción en línea denominada DigCompEdu Check-In, principal objeto de estudio de esta investigación (Cuartero et al., 2019; Gisbert, y Lázaro, 2016; Fernández-Márquez y Leiva-Olivencia, 2017; Tourón et al., 2018). En ella, se pretende conocer el nivel competencial digital del profesorado universitario de Andalucía (O1). Además, se trata de identificar si existen diferencias significativas entre la autopercepción de la CDD antes y después de realizar el cuestionario $(\mathrm{O} 2)$.

\section{Material y métodos}

Este trabajo se puede englobar en lo que se denominan investigaciones de metodología ex post facto, aquella que se aplica después de que el hecho haya sucedido, sin modificar las variables (Hernández, Fernández y Baptista, 2014). Se plantea un diseño de investigación transversal con enfoque descriptivo y contraste de hipótesis que tiene en cuenta la participación de profesores de todas las universidades públicas andaluzas.

\section{Muestra}

En la investigación participan 2262 docentes, 1026 hombres $(45.4 \%)$ y 1236 mujeres (54.6\%), la mayoría entre 50-54 años (37.3\%) y 666 entre $40-49$ años (29.4\%). Todos ellos trabajan como profesores universitarios en una de las 9 universidades públicas andaluzas.

En la Tabla 1 se presentan las áreas de conocimiento a la que pertenece cada profesor, así como sus años de experiencia.

Tabla 1.

Rama de conocimiento y años de experiencia de los profesores.

\begin{tabular}{llll}
\hline & & F & $\%$ \\
\hline \multirow{2}{*}{ Rama de } & Artes y Humanidades & 360 & 15.9 \\
conocimiento & Ciencias & 346 & 15.3 \\
& Ciencias de la Salud & 300 & 13.3 \\
& Ingeniería y Arquitectura & 434 & 19.2 \\
& Ciencias Sociales y Jurídicas & 822 & 36.3 \\
\hline \multirow{3}{*}{ Años de } & 1-3 años & 194 & 8.6 \\
experiencia & 4-5 años & 124 & 5.5 \\
docente & 6-9 años & 194 & 8.6 \\
& 10-14 años & 308 & 13.6 \\
& 15-19 años & 268 & 11.8 \\
& 20 o más años & 1174 & 51.9 \\
\hline
\end{tabular}

En la Tabla 2 se recogen los datos relativos al tiempo que los profesores llevaban utilizando las TIC en la enseñanza y el porcentaje de uso TIC en sus clases.

Tabla 2.

Años utilización de las tecnologías y tiempo dedicado a su uso.

\begin{tabular}{|c|c|c|c|}
\hline & & $\mathrm{F}$ & $\%$ \\
\hline \multirow{8}{*}{$\begin{array}{l}\text { Tiempo uso de las } \\
\text { tecnologías }\end{array}$} & $\begin{array}{l}\text { No uso la tecnología como } \\
\text { herramienta educativa }\end{array}$ & 34 & 1.5 \\
\hline & Menos de 1 año & 38 & 1.7 \\
\hline & 1-3 años & 202 & 8.9 \\
\hline & 4-5 años & 168 & 7.4 \\
\hline & 6-9 años & 312 & 13.8 \\
\hline & 10-14 años & 594 & 26.3 \\
\hline & 15-19 años & 466 & 20.6 \\
\hline & 20 años o más & 448 & 19.8 \\
\hline \multirow{8}{*}{$\begin{array}{l}\text { Porcentaje de tiem- } \\
\text { po dedicado al uso } \\
\text { de la tecnología en } \\
\text { clase }\end{array}$} & $0-10 \%$ & 172 & 7.6 \\
\hline & $11-25 \%$ & 572 & 25.3 \\
\hline & $26-50 \%$ & 674 & 29.8 \\
\hline & $51-75 \%$ & 542 & 24.0 \\
\hline & $76-100 \%$ & 302 & 13.4 \\
\hline & $0-10 \%$ & 172 & 7.6 \\
\hline & $11-25 \%$ & 572 & 25.3 \\
\hline & $26-50 \%$ & 674 & 29.8 \\
\hline
\end{tabular}


Como se puede observar, la gran mayoría de los encuestados lleva usando las tecnologías de la información y comunicación (TIC) como herramienta educativa durante bastante tiempo.

Entre los que indican que participan en un programa relacionado con la digitalización de los procesos de enseñanza y aprendizaje TIC ( $f=1156,51.1 \%$ ), los que no participan en los programas existentes en su centro $(\mathrm{f}=502,22.2 \%)$ y los que no disponen de tales programas $(\mathrm{f}=604,26.7 \%)$.

Por lo que se refiere a su competencia para el manejo de diferentes tecnologías (dominio tecnológico), las respuestas alcanzadas se presentan en la Tabla 3.

Tabla 3.

Competencias en el manejo de diferentes tecnologías

\begin{tabular}{lccccc}
\hline \multicolumn{5}{l}{ En mi día a día se manejar.... } \\
\hline Medio & T.d & E.d & N.d/N.a & D.a. & M.a. \\
\hline Ordenador & $84(3.7 \%)$ & $2(0.1 \%)$ & $22(1.0 \%)$ & $314(13.9 \%)$ & $1840(81.3 \%)$ \\
Tablet & $110(4.9 \%)$ & $16(0.7 \%)$ & $100(4.4 \%)$ & $434(19.2 \%)$ & $1602(70.8 \%)$
\end{tabular}

Smartphone $100(4.4 \%) 16(0.7 \%) \quad 102(4.5 \%) 412(18.2 \%) 1632(72.1 \%)$

Internet $\quad 84(3.7 \%) \quad 14(0.6 \%) \quad 90(4.0 \%) \quad 572(25.3 \%) 1502(66.4 \%)$

Nota: T.d.= Totalmente en desacuerdo; E.d= En desacuerdo; N.D/N. $\mathrm{a}=\mathrm{Ni}$ en desacuerdo ni en de acuerdo; D. $\mathrm{a}=$ De acuerdo; M. $\mathrm{a}=$ Muy de acuerdo.

Conjuntamente, hay que indicar que la gran mayoría de los profesores que cumplimentan el cuestionario sienten curiosidad por nuevas aplicaciones, programas y recursos digitales. En concreto, el 28,7\% ( $\mathrm{f}=650)$ estaba de acuerdo con la afirmación, y 1248 (55.2\%) muy de acuerdo, más del 80\% de la distribución. En oposición, 82 (3.6\%) está totalmente en desacuerdo y 44 (1.9\%) en desacuerdo. Solamente 238 profesores (10.5\%) no están ni de acuerdo ni en desacuerdo.

Por lo que se refiere a las redes sociales de las que son usuarios los profesores, en la Tabla 4 se presentan los resultados encontrados.

Tabla 4.

Número de redes sociales a las que el docente está suscrito

\begin{tabular}{lll}
\hline $\mathrm{N}^{\mathrm{o}}$ & $\mathrm{f}$ & $\%$ \\
\hline Ninguna & 118 & 5.2 \\
1 & 350 & 15.5 \\
2 & 486 & 21.5 \\
3 & 612 & 27.1 \\
4 & 312 & 13.8 \\
5 & 216 & 9.5 \\
Más de 6 & 168 & 7.4 \\
\hline
\end{tabular}

Tal y como reflejan los datos, más de la mitad de los encuestados son usuarios asiduos de más de 3 redes sociales (57.8\%).

\section{Instrumento de recogida de datos}

El instrumento de recogida de información fue el cuestionario "DigCompEdu Chek-in" (Cabero-Almenara y Palacios-Rodríguez, 2020), instrumento de análisis del Marco Europeo de Competencia Digital Docente DigCompEdu validado por Gho- mi \& Redecker (2018). Este marco competencial es seleccionado como el más adecuado para valorar la CDD del profesorado universitario mediante el juicio de expertos (Cabero-Almenara et al., 2020).

El instrumento está compuesto por veintidós ítems, que se refieren a las seis áreas competenciales que analiza DigCompEdu y que se presentaron anteriormente: a) compromiso profesional (cuatro ítems), b) recursos digitales (tres ítems), c) pedagogía digital (cuatro ítems), d) evaluación y retroalimentación (tres ítems), e) empoderar a los estudiantes (tres ítems) y f) facilitar la competencia digital de los estudiantes (cinco ítems).

Indicar que la primera área competencial es considerada por los autores del modelo la que hace referencia a la competencia profesional; las áreas recursos digitales, pedagogía digital, evaluación y retroalimentación y empoderar a los estudiantes, la competencia pedagógica; y la última, facilitar la competencia digital de los estudiantes, la competencia de los estudiantes.

Cada uno de los ítems mide las diferentes competencias que conforman el marco competencial: A. Comunicación organizacional; A2 Colaboración profesional; A3 Práctica reflexiva; A4 Desarrollo profesional Continuo Digital; B1 Selección de recursos digitales; B2 Creación y modificación de recursos digitales; B3 Administrar, proteger y compartir recursos digitales; C1 Enseñanza; C2 Guía; C3 Aprendizaje colaborativo; C4 Aprendizaje autodirigido; D1 Estrategias de evaluación; D2 Analizar pruebas; D3 Retroalimentación y planificación; E1 Accesibilidad e inclusión; E2 Diferenciación y personalización; E3 Participación activa de los estudiantes; F1 Información y alfabetización mediática; F2 Comunicación y colaboración digital; F3 Creación de contenido digital; F4 Bienestar; y F5 Solución digital de problemas.

Conjuntamente, en el cuestionario se le pide al docente que clasifique su nivel autopercibido de competencia digital: cuando comienza la elaboración del cuestionario (pre) y cuando la finaliza (post). El sistema de ordenación es el siguiente: Novato (A1), explorador (A2), integrador (B1), experto (B2), líder (C1) y pionero (C2).

Para la fiabilización del cuestionario se utiliza la Omega de McDonald (1999), pues presenta una serie de ventajas frente al tradicional Alfa de Cronbach: su resistencia a muestreos en poblaciones heterogéneas, no requerir el cumplir la tau-equivalencia y la ausencia de errores correlacionados (Domínguez-Lara y Merino-Soto, 2015; Ventura-León y Caycho-Rodríguez, 2017; Ventura-León, 2018). Los resultados son presentados en la Tabla 5.

Tabla 5.

Coeficientes de fiabilidad

\begin{tabular}{lc}
\hline Dimensión & $\alpha$ \\
\hline Compromiso profesional & .842 \\
Recursos digitales & .807 \\
Pedagogía digital & .821 \\
Evaluación y retroalimentación & .790 \\
Empoderar a los estudiantes & .784 \\
Facilitar la competencia digital de los estudiantes & .898 \\
\hline TOTAL & .967 \\
\hline
\end{tabular}

Todos los valores, de acuerdo con O'Dwyer y Bernauer (2014), denotan altos niveles de fiabilidad $(\alpha>.75)$, tanto para la globalidad del instrumento, como para las diferentes dimensiones que lo forman y las macro competencias a las que se refiere. 


\section{Procedimiento de recogida y análisis de datos}

El cuestionario se ha realizado en formato digital, a través de la plataforma "EuSurvey". La recogida de datos se llevó a cabo durante los dos primeros meses de 2020.

Se realizan análisis de tendencia central (media) y dispersión (desviación estándar). Además, se aplican estadísticos de contraste para hacer una comparación en las valoraciones iniciales y finales. En concreto, se ha utilizado la prueba no paramétrica "W de Wilkonxon" para valorar la magnitud de las diferencias. Paralelamente, se ha comprobado que los datos no se distribuyen normalmente a través del estudio de asimetría y curtosis. La prueba de "bondad de ajuste Kolmogorov-Smirnov" ha confirmado esta comprobación, con significación (p-valor) igual a .000 para todos los ítems (distribución no normal). En todo momento, los datos obtenidos son analizados con el paquete estadístico SPSS (V.26).

\section{Resultados}

Se debe tener en cuenta que, para una correcta interpretación de los resultados, el intervalo de respuesta oscila entre 0 y 4 . Por lo que se refiere a las puntuaciones medias, la Tabla 6 presenta los valores medios y las desviaciones estándar alcanzados por los profesores en las diferentes preguntas y en la globalidad de las áreas (O1).

Tabla 6.

Puntuaciones medias y desviación de los ítems y dimensiones del cuestionario

\begin{tabular}{lcc}
\hline & $\overline{\mathrm{x}}$ & $\sigma$ \\
\hline Área 1: Compromiso Profesional (A) & 2.28 & .77 \\
\hline
\end{tabular}

A1. Uso sistemáticamente diferentes canales digitales

para mejorar la comunicación con el alumnado y mis

compañeros/as. Por ejemplo: correos electrónicos, apli- $\quad 2.37 \quad .80$

caciones de mensajería tipo Whatsapp, blogs, el sitio

web de la facultad...

A2. Uso tecnologías digitales para trabajar con mis com-

pañeros/as dentro y fuera de mi organización educa- $2.20 \quad .93$

tiva.

A3. Desarrollo activamente mi competencia digital docente.

A4. Participo en cursos de formación online. Por ejemplo: cursos online de la universidad, MOOCs, webinars...

\begin{tabular}{lll}
\hline Área 2: Recursos Digitales (B) & 2.34 & .75 \\
\hline
\end{tabular}

B1. Utilizo diferentes sitios de internet (páginas web)

$\begin{array}{lll}\text { y estrategias de búsqueda para encontrar y seleccionar } & 2.30 \quad 96\end{array}$

una amplia gama de recursos digitales.

B2. Creo mis propios recursos digitales y modifico los

existentes para adaptarlos a mis necesidades como do- $\quad 2.50 \quad .89$

cente.

B3. Protejo el contenido sensible de forma segura. Por

ejemplo: exámenes, calificaciones, datos personales.

$2.22 \quad 1.14$

Área 3: Pedagogía Digital (C)

$3.26 \quad .92$

C1. Considero cuidadosamente cómo, cuándo y por qué usar las tecnologías digitales en clase, para garantizar $2.20 \quad 1.16$ que se aproveche su valor añadido.

C2. Superviso las actividades e interacciones de mis alumnos en los entornos de colaboración en línea que $\quad 2.30 \quad 1.13$ utilizamos.
C3. Cuando mis alumnos trabajan en grupos o equipos,

usan tecnologías digitales para adquirir y documentar 2.45

$\mathrm{o}$

conocimientos.

C4. Uso tecnologías digitales para permitir que los estudiantes planifiquen, documenten y evalúen su aprendizaje por sí mismos. Por ejemplo: pruebas de auto2.09 1.08 evaluación, portfolio digital, blogs, foros...

Área 4: Evaluación y Retroalimentación (D)

$1.98 \quad 1.02$

D1. Uso estrategias de evaluación digital para moni-

torizar el progreso de los estudiantes.

1.95

97

D2. Analizo todos los datos disponibles para identificar al alumnado que necesita apoyo adicional. "Datos" incluye: participación de los estudiantes, desempeño, calificaciones, asistencia, actividades e interacciones sociales en entornos en línea... El "alumnado que necesita apoyo adicional" es: aquel en riesgo de abandono escolar, bajo rendimiento, trastorno de aprendizaje, necesidades específicas de aprendizaje o que carece de habilidades transversales (habilidades sociales, verbales o de estudio).

D3. Uso tecnologías digitales para proporcionar retroalimentación (feedback) efectiva.

Área 5: Empoderar a los Estudiantes (E)

E1. Cuando propongo tareas digitales, considero y abordo posibles problemas como el acceso igualitario a los dispositivos y recursos digitales; problemas de compatibilidad o nivel bajo de competencia digital del alumnado.

E2. Uso tecnologías digitales para ofrecer al alumnado oportunidades de aprendizaje personalizadas. Por ejemplo: asignación de diferentes tareas digitales para abordar las necesidades de aprendizaje individuales, tener en cuenta las preferencias e intereses...

E.3 Uso tecnologías digitales para que el alumnado participe activamente en clase.

Área 6: Facilitar la Competencia Digital de los Estudiantes (F)

$1.94 \quad .85$

F1. Enseño al alumnado cómo evaluar la confiabilidad de la información buscada en línea y a identificar información errónea y/o sesgada.

F2. Propongo tareas que requieren que los estudiantes usen medios digitales para comunicarse y colaborar en- 2.02 tre sí o con una audiencia externa.

F3. Propongo tareas que requieren que los estudiantes creen contenido digital. Por ejemplo: videos, audios, fo- 2.28 tos, presentaciones, blogs, wikis...

F4. Enseño al alumnado cómo comportarse de manera segura y responsable en línea.

F5. Animo al alumnado a usar las tecnologías digitales de manera creativa para resolver problemas concretos. Por ejemplo, superar obstáculos o retos emergentes en su proceso de aprendizaje.

Los valores medios alcanzados por los docentes en la globalidad del instrumento fueron de 2.11 con una desviación típica de 0.74 . Valor que nos señala que los profesores se han posicionado en 
un valor central. Esto nos lleva a señalar que, en general, la percepción que tienen de su dominio de las CDD es moderada. Además, ordenandos de menor a mayor, los resultados por dimensiones son los siguientes: Área 5: Empoderar a los Estudiantes (1.94); Área 4: Evaluación y Retroalimentación (1.98); Área 6: Facilitar la Competencia Digital de los Estudiantes (2.11); Área 1: Compromiso Profesional (2.28); Área 2: Recursos Digitales (2.34); Área 3: Pedagogía Digital (3.26). Valores que nos permiten señalar que, en general, los docentes presentan niveles bajos de CDD; si bien se consideran moderadamente competentes en compromiso profesional y en recursos digitales. Es significativo que la puntuación más elevada se ha desarrollado en la competencia "Pedagogía Digital".

La Tabla 7 presenta los resultados encontrados tanto en la primera como en la segunda valoración del nivel competencial percibido.

Tabla 7.

Valoración por parte del docente

\begin{tabular}{rcccc}
\hline \multirow{2}{*}{ Nivel } & \multicolumn{2}{c}{ Pre } & \multicolumn{2}{c}{ Pos } \\
\cline { 2 - 5 } & $\mathbf{f}$ & \% & f & $\%$ \\
\hline Novato & 66 & 2.9 & 74 & 3.3 \\
Explorador & 390 & 17.2 & 390 & 17.2 \\
Integrador & 944 & 41.7 & 976 & 43.1 \\
Experto & 616 & 27.2 & 590 & 26.1 \\
Líder & 170 & 7.5 & 174 & 7.7 \\
Pionero & 76 & 3.4 & 58 & 2.6 \\
\hline
\end{tabular}

Antes de realizar la prueba, la mayoría de los profesores se sitúan en el nivel integrador (68.9\%) mientras que, tras contestar el cuestionario, hay un aumento del $1.4 \%$ en este nivel. Esta misma tendencia puede ser observada en los niveles novato $(+0.4 \%)$ y líder (+0.2). El resto de niveles mantienen o disminuyen los porcentajes respecto a la primera valoración: explorador $(=)$, experto $(-1.1 \%)$ y pionero $(-0.8 \%)$.

Con objeto de analizar si estas diferencias entre la primera y segunda valoración son significativas $(\mathrm{O} 2)$, se formulan las siguientes hipótesis:

- Hipótesis nula (H0): No existen diferencias significativas entre las valoraciones que realiza el docente antes y después de cumplimentar le cuestionario.

- Hipótesis alternativa (H1): Existen diferencias significativas entre las valoraciones que realiza el docente antes y después de cumplimentar le cuestionario.

Para ello se aplica el estadístico no paramétrico de prueba de rangos con signos de Wilcoxon. La Tabla 8 presenta los valores de rango positivo y el valor alcanzado.

Tabla 8.

Prueba de rangos con signos de Wilcoxon

\begin{tabular}{lccccc}
\hline & $\mathrm{N}$ & $\begin{array}{c}\text { Rango } \\
\text { promedio }\end{array}$ & $\begin{array}{c}\text { Suma de } \\
\text { rangos }\end{array}$ & Wilcoxon & Significación \\
\hline $\begin{array}{l}\text { Rangos } \\
\text { negativos }\end{array}$ & $328 \mathrm{a}$ & 296,68 & 97312,00 & -3270 & .001 \\
$\begin{array}{l}\text { Rangos } \\
\text { positivos }\end{array}$ & $256 \mathrm{~b}$ & 287,14 & 73508,00 & & \\
Empates & $1678 \mathrm{c}$ & & & & \\
\hline
\end{tabular}

En base a estos valores, se puede afirmar con un 99\% de confianza que existen diferencias significativas entre la autopercepción del docente antes y después de cumplimentar le cuestionario. En este caso, las puntuaciones otorgadas antes de realizar el cuestionario son superiores a las asignadas una vez se ha contestado y reflexionado sobre la CDD.

\section{Discusión}

Debido al alto número de profesores encuestados, la descripción de la muestra permite hacer una panorámica del perfil del docente universitario andaluz. En general, profesores con más de 10 años de experiencia que lleva usando las tecnologías de la información y comunicación (TIC) como herramienta educativa durante bastante tiempo. Estos resultados coinciden con los presentados por Krumsvik et al. (2016) y García-Marco et al. (2020). Sin embargo, hay que tener en cuenta que la variable experiencia aparece como reflejo de la disminución en el nivel de competencia en el estudio de Garzón et al. (2020), donde los profesores más jóvenes están más interesados en la formación competencial. Conjuntamente, la autopercepción de dominio tecnológico (ordenador, tablet, smartphone, internet...) es alta y son usuarios asiduos de más de tres redes sociales. Destacar que el número de redes sociales de las que es usuario el profesorado es un buen indicador en el desarrollo de la competencia digital, evidenciando las aportaciones de García-Pérez et al. (2016), Porlan y Sanchez (2016), Eyo (2016) y Sánchez-Gómez et al. (2017).

Uno de los principales objetivos del estudio consiste en identificar el nivel de competencia digital docente del profesorado universitario andaluz (O1). Los resultados muestran que, en general, el nivel es moderado. En concreto, las áreas mejor valoradas han sido las de Pedagogía Digital, relacionada con saber diseñar y planificar el uso de las tecnologías en el aprendizaje del alumnado con metodologías activas; y Recursos Digitales, afín con la creación y distribución de recursos digitales en el aula, respetando las normas de derechos de autor. Estos hallazgos inducen a pensar, al igual que Pinto et al. (2017) y Gómez-Triguero, Ruiz-Bañuls y Ortega-Sánchez (2019), que el profesorado transita de una concepción digital centrada en el uso de recursos TIC, a concepciones más amplias como la creación de conocimiento a través de las TAC y participación en entornos colaborativos a través de las TEP. Así mismo, las áreas con las puntuaciones más bajas son las de Empoderar a los Estudiantes, relacionada con asegurar el acceso digital a todo el alumnado, ofrecer actividades de aprendizaje adaptadas a su nivel competencial, así como a sus intereses y necesidades educativas; y Evaluación y Retroalimentación, enfocada en el uso de recursos TIC para la evaluación, retroalimentación y mejora del rendimiento del alumnado. En ese sentido, está claro que es necesaria una formación competencial del profesorado, puesto que un dominio deficiente de la misma lleva implícito una educación no enfocada en las profesiones más demandadas del siglo XXI (Padilla-Hernández et al., 2020).

Por otro lado, se ha demostrado que las puntuaciones otorgadas antes de realizar el cuestionario son superiores a las asignadas una vez se ha contestado y reflexionado sobre la CDD $(\mathrm{O} 2)$. Este hecho puede ser explicado por una idealización de la autopercepción competencial. El docente posee una serie de modelos mentales relacionados con su propia competencia digital (autopercepción idealista) que, tras contestar y reflexionar cumplimentando el cuestionario, cambian (autopercepción realista). Por este motivo, este tipo de herramientas suponen una "transformación de las estructuras, métodos y supuestos tradicionales comunicativos, educativos y laborales de los docentes" (Cabero-Almenara y Palacios-Rodríguez, 2020, p.16). 
Estos hallazgos ponen de manifiesto la necesidad de llevar a cabo planes personalizados de formación docente que permitan situarse en niveles competenciales avanzados, como aquellos centrados en la innovación y liderazgo pedagógico con TIC.

\section{Referencias}

Aslan, A. \& Zhu, Ch. (2016). Influencing Factors and Integration of ICT into Teaching Practices of Pre-service and Starting Teachers. International Journal of Research in Education and Science, 2(2), 359-370. https:/ / files.eric.ed.gov/fulltext/EJ1105123.pdf

Bahçivan, E., Güneş, E., \& Üstündağ, M. (2018). A comprehensive model covering prospective teachers' technology use: the relationships among self, teaching and learning conceptions and attitudes. Technology, Pedagogy and Education, 27(4), 399-416. https://doi.org/10.1080/1475939X.2018.1479296.

Beneyto-Seoane, M., \& Collet-Sabé, J. (2018). Análisis de la actual formación docente en competencias TIC. Por una nueva perspectiva basada en las competencias, las experiencias y los conocimientos previos de los docentes. Profesorado. Revista de Currículum y Formación Del Profesorado, 4(23), 45-57. https: / / doi. org/10.30827/profesorado.v22i4.8396

Bernal, C., Gozávez, V., Hernando A., \& Masanet, M.J. (2019). Relación entre formación universitaria y competencia mediática del profesorado. Revista Complutense de Educación, 30(4), 11131126. https:/ /dx.doi.org/10.5209/rced.60188.

Blau, I., \& Shamir-Inbal, T. (2017). Digital competences and long-term ICT integration in school culture: The perspective of elementary school leaders. Education and Information Technologies, 22(3), 769-787. https:/ / doi.org/10.1007/s10639-015-9456-7

Cabero, J., \& Martínez, A. (2019). Las tecnologías de la información y comunicación y la formación inicial de los docentes. Modelos y competencias digitales. Profesorado. Revista de $\mathrm{Cu}$ rriculum y Formación del Profesorado, 23(3), 247-268. https:/ /doi. org/10.30827/profesorado.v23i3.9421

Cabero-Almenara, J., \& Palacios-Rodríguez, A. (2020). Marco europeo de Competencia Digital Docente «DigCompEdu». Traducción y adaptación del cuestionario «DigCompEdu CheckIn». EDMETIC, 9(1), 213-234. https://doi.org/10.21071/edmetic.v9i1.12462

Cabero-Almenara, J., Barroso-Osuna, J., Palacios-Rodríguez, A., \& Llorente-Cejudo, C. (2020). Marcos de Competencias Digitales para docentes universitarios: su evaluación a través del coeficiente competencia experta. Revista Electrónica Interuniversitaria de Formación del Profesorado, 23(2). https: / / doi.org/10.6018/ reifop. 413601

Cabero-Almenara, J., Estrada-Vidal, L., \& Gutiérrez-Castillo, J. J. (2017). Diseño y validación de un instrumento de evaluación de la competencia digital del estudiante universitario. Revista Espacios, 38(10), 16. https://doi.org/10.11144/Javeriana.m8-17. ctap

Cela-Ranilla, J. M., Esteve González, V., Mon, F., González Martínez, J., \& Gisbert-Cervera, M. (2017). El docente en la sociedad digital: Una propuesta basada en la pedagogía transformativa y en la tecnología avanzada. Profesorado, Revista de curriculum y formación del profesorado, 21(1), 403-422. https: / / recyt.fecyt.es / index.php/profesorado/article/view/58073/35585

Centro Común de Investigación de la Unión Europea (JCR) (2018). ESHorizonte2020. Recuperado de https://eshorizonte2020.es/mas-europa / centro-comun-de-investigacion-jrc

Choi, M., Cristol, D., \& Gimbert, B. (2018). Teachers as digital citizens: The influence of individual backgrounds, internet use and psychological characteristics on teachers' levels of digital citizen-ship. Computers y Education, 121, 143-161. https://doi. org/10.1016/J.COMPEDU.2018.03.005

Comisión de las Comunidades Europeas. (2003). Educación y Formación 2010. Urgen las reformas para coronar con éxito la estrategia de Lisboa. Publications Office of the European Union.

Comisión Europea (2012). Un nuevo concepto de educación: invertir en las competencias para lograr mejores resultados socioeconómicos. Publications Office of the European Union.

Consejería de Educación y Deporte. (2018). \#PRODIG. Programa de Transformación Digital de Centros. Dirección General de Formación Profesorado e Innovación Educativa.

Consejo de la Unión Europea. (2003). Conclusiones de la Mesa. Consejo Europeo de Bruselas de 20 y 21 de marzo de 2003. Publications Office of the European Union.

Consejo de la Unión Europea. (2018). Recomendación del Consejo, de 22 de mayo de 2018, relativa a las competencias clave para el aprendizaje permanente. Diario Oficial de la Unión Europea.

Cuartero, M., Espinosa, M., \& Porlán, I. (2019). Certificación de la Competencia Digital Docente: propuesta para el profesorado universitario. RIED. Revista Iberoamericana de Educación a Distancia, 22(1), 187-205. https:/ / doi.org/10.5944/RIED.22.1.22069

Cuartero, M., Porlán, I., \& Espinosa, M. (2016). Análisis Conceptual de Modelos de Competencia Digital del Profesorado Universitario. RELATEC. Revista Latinoamericana de Tecnología Educativa, 15(1), 97-114. https:/ / doi.org/10.17398/1695-288X.15.1.97

Domínguez-Lara, S., \& Merino-Soto, C. (2015). Why is it important to report the confidence intervals of Cronbach's alpha coefficient? Revista Latinoamericana de Ciencias Sociales, Niñez y Juventud, 13, 1326-1328.

Durán, M., Gutiérrez, I., \& Prendes, M. (2016). Análisis conceptual de modelos de competencia digital del profesorado universitario. RELATEC. Revista Latinoamericana de Tecnología Educativa, 15(1), 97-114. https:/ / doi.org/10.17398/1695-288X.15.1.97

Eyo, M. (2016). Counselling implications of teachers' digital competencies in the use of Social Networking Sites (SNSs) in the teaching-learning process in Calabar, Nigeria. Cogent Education, 3(1), 1-9. https:/ / doi.org/10.1080/2331186X.2015.1128134

Fernández-Márquez, E., Leiva-Olivencia, J., \& López-Meneses, E. (2017). Competencias digitales en docentes de Educación Superior. Revista de Digital de Investigación en Docencia Universitaria, 12(1), 213-231. http:/ / dx.doi.org/10.19083/ridu.12.558

Fernández-Márquez, E., \& Leiva-Olivencia, J. (2017). Competencias digitales en docentes de Educación Superior Digital Competences in Higher Education Professors. Revista Digital de Investigación en Docencia Universitaria, 12(2), 213-231. https:// doi.org/10.19083/ridu.12.558

Flores-Lueg, C., \& Roig Vila, R. (2016). Diseño y validación de una escala de autoevaluación de competencias digitales para estudiantes de pedagogía. Píxel-Bit. Revista de Medios y Educación, 48, 209-224. https:/ /doi.org/10.12795/pixelbit.2016.i48.14

García Tartera, F. (2016). Competencias digitales en la docencia universitaria del siglo XXI. Universidad Complutense de Madrid.

García-Marcos, C., López-Vargas, O., \& Cabero-Almenara, J. (2020). Autorregulación del aprendizaje en la Formación Profesional a Distancia: efectos de la gestión del tiempo. Revista de Educación a Distancia, 20(62), 1-21. https://doi.org/10.6018/ red.400071

García-Perez, R., Rebollo-Catalán, A., \& García-Perez, C. (2016). The relationship between teacher training preferences and their digital skills on social networks. BORDON. Revista de Pedagogía, 68(2), 137-153.

García-Valcárcel Muñoz-Repiso, A., Basilotta Gómez-Pablos, V., Cabezas González, M., Casillas Martín, S., González Rodero, L., Hernández Martín, A., \& Mena Marcos, J. (2015). La forma- 
ción del profesorado universitario en Tecnologías de la Información y la Comunicación en la Universidad de Salamanca. RELATEC: Revista Latinoamericana de Tecnología Educativa, 14(1), 75-88. https: / / doi.org/10.17398/1695-288X.14.1.75

Garzón, E., Sola, T., Ortega, J., Marín, J., \& Gómez, G. (2020). Teacher Training in Lifelong Learning. The Importance of Digital Competence in the Encouragement of Teaching Innovation. Sustainability, 12, 28-52. https://doi.org/10.3390/su12072852

Ghomi, M., \& Redecker, C. (2018). Digital Competence of Educators (DigCompEdu): Development and Evaluation of a Self-Assessment Instrument for Teachers' Digital Competence. Joint Re-search Center.

Ghomi, M., \& Redecker, C. (2019). Digital Competence of Educators (DigCompEdu): Development and Evaluation of a Self-assessment Instrument for Teachers' Digital Competence. En Proceedings of the 11th International Conference on Computer Supported Education (Vol. 1, pp. 541-548). SCITEPRESS - Science and Technology Publications. https://doi. org/10.5220/0007679005410548

Gil-Flores, J., Rodríguez-Santero, J., \& Torres-Gordillo, J.-J. (2017). Factors that explain the use of ICT in secondary-education classrooms: The role of teacher characteristics and school infrastructure. Computers in Human Behavior, 68, 441-449. https:/ / doi.org/10.1016/J.CHB.2016.11.057

Gisbert, M., \& Lázaro, J. (2015). Professional development in teacher digital competence and improving school quality from the teachers' perspective: a case study. New approaches in educational research, 4(2), 124-131. https://doi.org/10.7821/ naer.2015.7.123

Gómez-Parra, M. E., \& Huertas-Abril, C. (2019). La importancia de la competencia digital para la superación de la brecha lingüística en el siglo XXI: Aproximación, factores y estrategias. EDMETIC, 8(1). https://doi.org/10.21071/edmetic.v8i1.11095

Gómez-Triguero, I., Ruiz-Bañuls, M., \& Ortega-Sánchez, D. (2019). Digital Literacy of Teachers in Training: Moving from ICTs (Information and Communication Technologies) to LKTs (Learning and Knowledge Technologies). Education Sciences, 9, 274, 1-10. https / / doi.org/10.3390/educsci9040274

Hatlevik, O., Guðmundsdóttir, G., \& Loi, M. (2015). Digital diversity among upper secondary students: a multilevel analysis of the relationship between cultural capital, self-efficacy, strategic use of information and digital competence. Computers $\mathcal{E}$ Education, 81, 345-353. https://doi.org/10.1016/j.compedu.2014.10.019

Hatlevik, O., Throndsen, I., Loi, M., \& Gudmundsdottir, G. (2018). Students' ICT self-efficacy and computer and information literacy: Determinants and relationships. Computers $\mathcal{E}$ Education, 118, 107-119. https:/ / doi.org/10.1016/J.COMPEDU.2017.11.011

Hernández, R., Fernández, C., \& Baptista, P. (2014). Metodología de la investigación. McGraw-Hill Education.

Hilliger, I., Ortiz-Rojas, M., Pesántez-Cabrera, P., Scheihing, E., Tsai, Y. S., Muñoz-Merino, P. J., \& Pérez-Sanagustín, M. (2020). Identifying needs for learning analytics adoption in Latin American universities: A mixed-methods approach. Internet and Higher Education, 45, 100726. https://doi.org/10.1016/j.iheduc.2020.100726

INTEF. (2017a). Comparativa Marco Competencia Digital Docente INTEF 2017 (versión octubre 2017) y Marco Europeo DigCompEdu JRC (versión diciembre 2017). Instituto Nacional de Tecnologías Educativas y Formación del Profesorado.

INTEF. (2017b). Marco Común de Competencia Digital Docente. Instituto Nacional de Tecnologías Educativas y Formación del Profesorado.
Kluzer, S., Pujol Priego, L., Carretero, S., Punie, Y., Vuorikari, R., Cabrera, M. \& Okeeffe, W. (2018) DigComp into action, get inspired make it happen a user guide to the European Digital Competence framework. JRC. https:/ / doi.org/10.2760/112945

Krumsvik, R., Jones, L., Øfstegaard, M., \& Eikeland, O. (2016). Upper secondary school teachers' digital competence: Analysed by demographic, personal and professional characteristics. Nordic Journal of Digital Literacy, 11(03), 143-164.

Lázaro, J., Usart, \& Gisbert, M. (2019). Assessing Teacher Digital Competence: the Construction of an Instrument for Measuring the Knowledge of Pre-Service Teachers. Journal of New Approaches in Educational Research, 8(1), 73-78. https:/ / doi. org/10.7821/naer.2019.1.370.

Marín-Díaz, V., Reche, E., \& Maldonado, G. (2013). Ventajas e inconvenientes de la formación online. RIDU. Revista Digital de Investigación en Docencia Universitaria, 7(1), 33-43. https://revistas.upc.edu.pe/index.php/docencia/article/view/185/141

McDonald, R. (1999). Test Theory: A Unified Treatment. Erlbaum

O'Dwyer, L., \& Bernauer, J. (2014). Quantitative research for the qualitative researcher. SAGE.

OpenMind BBVA (2017). La era de la perplejidad. Repensar el mundo que conocíamos. Taurus.

Padilla-Hernández, A., Gámiz-Sánchez, V., \& Romero-López, M. (2020). Evolution of higher education teachers' digital competence: Critical incidents derived from life stories. Educar, 56(1), 109-127. https://doi.org/10.5565/rev/educar.1088

Peñalva Vélez, A., Fraile, M., \& Lacambra, A. M. M. (2018). Competencia digital y alfabetización digital de los adultos (profesorado y familias). International Journal of New Education, 1(1). https:/ /doi.org/10.24310/IJNE1.1.2018.4892

Pinto, A., Cortés, O., \& Alfaro, C. (2017). Hacia la transformación de la práctica docente: modelo espiral de competencias TICTACTEP. Pixel-Bit. Revista de Medios y Educación, 51, 37-51 http:/ / dx.doi.org/10.12795/pixelbit.2017.i51.03

Porlan, I., \& Sanchez, J. (2016). Evaluation and development of digital competence in future primary school teachers at the University of Murcia. Journal of New Approaches in Educational Research, 5(1), 51-56.

Pozos, K. \& Tejada, J. (2018). Competencias digitales docentes en educación superior: niveles de dominio y necesidades formativas. Revista Digital de Investigación en Docencia Universitaria, 12(2), 59-87. doi: http:/ / dx.doi.org/10.19083/ridu.2018.712.

Redecker, C., \& Punie, Y. (2017). Digital Competence of Educators DigCompEdu. Publications Office of the European Union.

Rodríguez-García, A., Raso, F., \& Ruiz-Palmero, J. (2019). Competencia digital, educación superior y formación del profesorado: un estudio de meta-análisis en la web of science. Pixel-Bit. Revista de Medios y Educación, 54(4), 65-81. https:/ / doi. org/10.12795/pixelbit.2019.i54.04

Roig-Vila, R., Mengual-Andrés, S., y Quinto-Medrano, P. (2015). Conocimientos tecnológicos, pedagógicos y disciplinares del profesorado de Primaria. Comunicar, 45(23), 151-159. https:/ /doi.org/http://dx.doi.org/10.3916/C45-2015-16

Ruíz Mezcua, A. (2019). Competencia digital y TICs en interpretación: «renovarse o morir». EDMETIC, 8(1). https:/ / doi. org/10.21071/edmetic.v8i1.11062

Scherer, R., \& Siddiq, F. (2019). The relation between students' socioeconomic status and ICT literacy: Findings from a meta-analysis. Computers $\mathcal{E}$ Education, 138, 13-32. https://doi. org/10.1016/J.COMPEDU.2019.04.011

Solís de Ovando Calderón, J., \& Jara Jara, V. (2019). Competencia digital de docentes de Ciencias de la Salud de una universidad chilena. Pixel-Bit, Revista de Medios y Educación, 56, 193-211. https:/ / doi.org/10.12795/pixelbit.2019.i56.10 
Tourón, J., Martín, D., Navarro, E., Pradas, S., \& Íñigo, V. (2018). Validación de constructo de un ins-trumento para medir la competencia digital docente de los profesores (CDD). Revista Española de Pedagogía, 76(269), 25-54. https://doi.org/10.22550/ REP76-1-2018-02

Uerz, D., Volman, M., \& Kral, M. (2018). Teacher educators' competences in fostering student teachers' proficiency in teaching and learning with technology: An overview of relevant research literature. Teaching and Teacher Education, 70, 12-23. https://doi.org/10.1016/j.tate.2017.11.005.

UNESCO (2004). Las tecnologías de la información y la comunicación en la formación docente. Guía de planificación. UNESCO.

Ventura-León, J., \& Caycho-Rodríguez, T. (2017). Confidence Intervals for Omega Coefficient: Proposal for Calculus. Revista Latinoamericana de Ciencias Sociales, Niñez y Juventud, 15, 625-627.
Ventura-León. J. (2018). Confidence intervals for Omega coefficient: Proposal for calculation. Adicicones, 30, 77-78. https: / / pdfs.semanticscholar.org/b42c/821944974bc048aaf6c83019b2a0a8b4973e.pdf

Williamson, B., Potter, J., \& Eynon, R. (2019). New research problems and agendas in learning, media and technology: the editors' wishlist. Learning, Media and Technology, 44(2), 87-91. https://doi.org/10.1080/17439884.2019.1614953

Wilson, C., Grizzle, A., Tuazon, R., Akyempong, K., \& Cheung, C. (2011). Alfabetización mediática e informacional. Curriculum para profesores. UNESCO. http://unesdoc.unesco.org/images/0021/002160/216099S.pdf 
